\title{
Sexually antagonistic epigenetic marks that canalize sexually dimorphic development
}

\author{
William Rice, Urban Friberg and Sergey Gavrilets
}

\section{Linköping University Post Print}

\section{Tweet}

N.B.: When citing this work, cite the original article.

Original Publication:

William Rice, Urban Friberg and Sergey Gavrilets, Sexually antagonistic epigenetic marks that canalize sexually dimorphic development, 2016, Molecular Ecology, (25), 8, 1812-1822.

http://dx.doi.org/10.1111/mec. 13490

Copyright: Wiley: 12 months

http://eu.wiley.com/WileyCDA/

Postprint available at: Linköping University Electronic Press

http://urn.kb.se/resolve?urn=urn:nbn:se:liu:diva-128119 
SPECIAL ISSUE: EPIGENETICS

\section{Sexually antagonistic epigenetic marks that canalize sexually dimorphic development}

William R. Rice*, U. Friberg ${ }^{\ddagger}$, and Sergey Gavrilets ${ }^{\ddagger}$

*Department of Ecology, Evolution \& Marine Biology, University of California,

Santa Barbara, CA 93106 USA

† IFM Biology, AVIAN Behavioural Genomics and Physiology Group

Linköping University, SE-581 83 Linköping, Sweden

urban.friberg@liu.se

‡ Department of Ecology and Evolutionary Biology and Department of Mathematics, National Institute for Mathematical and Biological Synthesis, University of Tennessee, Knoxville, TN 37996, USA 37996, USA

Corresponding author:

William R. Rice

rice@lifesci.ucsb.edu

\section{KeyWords:}

sexual dimorphism, canalization, sexual conflict, gonad-trait discordance, epigenetics, sex-specific epimarks, transgenerational epigenetics 


\section{Abstract}

The sexes share the same autosomal genomes yet sexual dimorphism is common due to sex-specific gene expression. When present, $\mathrm{XX}$ and $\mathrm{XY}$ karyotypes trigger alternate regulatory cascades that determine sex-specific gene expression profiles. In mammals, secretion of testosterone $(T)$ by the testes during fetal development is the master switch influencing the gene expression pathways (male vs. female) that will be followed, but many genes have sex-specific expression prior to T secretion. Environmental factors, like endocrine disruptors and mimics, can interfere with sexual development. However, sex-specific ontogeny can be canalized by the production of epigenetic marks (epimarks) generated during early ontogeny that increase sensitivity of $X Y$ embryos to $T$ and decrease sensitivity of $X X$ embryos. Here we integrate and synthesize the evidence indicating that canalizing epimarks are produced during early ontogeny. We will also describe the evidence that such epimarks sometimes carry over across generations and produce mosaicism in which some traits are discordant with the gonad. Such carryover epimarks are sexually antagonistic because they benefit the individual in which they were formed (via canalization) but harm opposite-sex offspring when they fail to erase across generations and produce gonad-trait discordances. SA-epimarks have the potential to: i) magnify phenotypic variation for many sexually selected traits, ii) generate overlap along many dimensions of the masculinity/femininity spectrum, and iii) influence medically important gonad-trait discordances like cryptorchidism, hypospadias, and idiopathic hirsutism. 


\section{Introduction}

Sexual conflict within the genome is divided into two major categories (Arnqvist and Rowe 2005; Rice and Gavrilets 2014). Intra-locus conflict occurs when the optimal allele at a locus differs between the sexes, causing adaptive evolution by one sex to be at the fitness expense of the other sex (Haldane 1926; Mandel 1971). Allelomorphs with opposing effects in the two sexes are referred to as sexually antagonistic alleles (Rice 1984). The second category of sexual conflict is called inter-locus conflict (Parker 1979; Chapman et al. 1995; Arnqvist and Rowe 1995; Rice 1996; Rice and Holland 1997; Gavrilets et al. 2001). Here, alleles at different gene loci affect a shared interaction trait, with one locus influencing the female component of the trait and the other locus influencing the male component. Males and females usually interact in the context of a dyad to determine the outcome of the interaction trait (e.g., the decisions of whether or not to mate or whether each individual mates monogamously or promiscuously) and the optimal outcome of the interaction differs between the sexes.

Epigenetics creates the opportunity for a new and fundamentally different form of sexual conflict based epigenetic rather than genetic variation: sexually antagonistic epigenetic conflict (Rice et al. 2012). The foundation for this conflict is a sex-specific epigenetic mark that sometimes fails to erase across generations. For the conflict to be manifest, the sex-specific epigenetic marks (i.e., DNA methylation and/or histone tail modification, hereafter called "epimarks") must be an integral part of a sexually dimorphic ontogenetic process, that function to masculinize or feminize somatic gene expression (and the organismal-level phenotypes that they influence) during ontogeny. 
Most such epimarks will be absent in the germ line because they first appear too late in ontogeny to be shared between the soma and germ line via a coalescent mitotic lineage, and hence will not be carried-over trans-generationally. However, this barrier is absent for sex-specific epimarks that are produced prior to the separation of cell lineages between the germ line and soma, e,g., in embryonic stem cells (ESCs).

In mammals, the function of these embryonic, sex-specific epimarks is to control sexually dimorphic development prior to the secretion of testosterone $(T)$ by the testes, and/or to canalize (i.e., resist change in response to environmental and genetic perturbations) sex-specific ontogenetic responses to sex steroids. Most sexual dimorphism in mammals, however, is a response to fetal androgen signaling (fetal estrogen levels have little or no effect on mammalian sexual dimorphism), as well as androgen or estrogen signaling that commences at puberty (Thorton et al. 2009, and more fully described below). Because of this overarching influence of sex hormone signaling in the ontogeny of sexual dimorphism in mammals, we will focus here on sexspecific epimarks in the early embryo that canalize down-stream, sexually dimorphic ontogeny that occurs in response to sex steroid signaling, e.g., those epimarks that reduce the impact of natural variation in circulating sex steroid levels and/or environmental endocrine disruptors and steroid mimics on sexually dimorphic development. Later in the discussion, we will consider SA-epimarks that operate outside the context of sex hormone signaling, as well as sex-specific epimarks that are produced in parallel in both the germ line and the soma during fetal development. Conflict occurs when these epigenetic marks: i) fail to erase across generations, and ii) 
are inherited by opposite-sex offspring and influence their ontogeny. Such an epimark increases Darwinian fitness of the parent where it originated because it canalizes the parent's sexual development, but it reduces fitness in opposite-sex offspring by contributing to discordance between the gonadal sex and a sexually dimorphic trait. We will refer to the epimarks causing this epigenetic conflict as sexually antagonistic epigenetic marks -abbreviated as SA-epimarks (Rice et al. 2012, 2013).

The details of the formation, molecular composition, expression, and transmission of SA-epimarks are expected to differ between taxonomic groups because the types and relative importance of different kinds of epigenetic marks differ substantially among taxa. Here we will focus on mammals as a model taxa owing to the extensive epigenetic research that has been done on this group. However, the logic of SA-epigenetic marks that we develop in mammals should be applicable, in a general sense, to other groups.

We have previously detailed the evidence for the existence of SA-epimarks in the context of human sexual orientation (Rice et al. 2012) and we have also described a general protocol to screen for the causative epimarks using embryonic and hair-follicle stem cells (Rice et al. 2013). Here we focus on the broader context SA-epimarks influencing any sexually dimorphic trait. At this time, we have not identified and characterized specific SA-epimarks. Nonetheless, just as Watson and Crick (1953a,b) combined many disparate pieces of information to support a hypothesis for the structure and semiconservative replication of DNA -that motivated pivotal tests of this hypothesis, 
like that of Meselson and Stahl (1958)- our approach here is to follow the logic of these exemplary studies by integrating the many disparate lines of evidence that SA-epimarks contribute to a new and unappreciated form of sexual conflict.

\section{Requisite conditions for SA-epimarks}

The existence of SA-epimarks, that canalize sex-specific ontogeny in response to sex hormone signaling (Figure 1), is strongly motivated only if a well-defined set of requisite conditions is met, which we enumerate in detail below. In short, sexually dimorphic ontogeny must be canalized, the canalization must be advantageous due to developmental ambiguities, epimarks must contribute to the canalization and sometimes carry over across generations to opposite-sex offspring, and mutations coding for the epimarks must accumulate in response to selection.

1) Sexually dimorphic development is canalized. In mammals, this would require evidence that i) $X X$ fetuses have reduced sensitivity to elevated $T$, and $X Y$ fetuses have increased sensitivity to elevated $\mathrm{T}$, and ii) starting at puberty, $\mathrm{XY}$ males have increased sensitivity to $T$ and decreased sensitivity to estrogens, vice versa for $\mathrm{XX}$ females.

2) There is need (i.e., a fitness advantage) for canalization of sexually dimorphic development. If there is no fitness benefit in the parent that produced them, then carryover epimarks produce no sexual antagonism. In mammals, this would require 
evidence for sufficient ambiguity in i) fetal androgen signaling, and/or ii) androgen or estrogen signaling after puberty, to make canalizing epimarks advantageous. Sexual ambiguity in sex hormone signaling would be expected to arise due to intrinsic variation in sex-specific levels of sex hormones and/or environmental endocrine disruptors and androgen and estrogen mimics.

3) Sex-specific epimarks produced during early ontogeny influence down-stream sexually dimorphic development, i.e. there are sex-specific epimarks that are produced sufficiently early in ontogeny that they can canalize sexually dimorphic development later in ontogeny. In mammals, this would require evidence that sexspecific epimarks are produced prior the onset of testicular production of $\mathrm{T}$ for traits that become sexually dimorphic during later fetal development.

4) Some epigenetic marks are shared by both the soma and the germ line, i.e., they are mitotically inherited by both cell lineages from a common progenitor cell(s), or they are produced in parallel in both cell lineages. In mammals this would require evidence that sex-specific epimarks are produced in embryonic stem cells before the separation of soma and germ line, and/or that sex-specific epimarks produced later in development are generated in parallel in both the soma and germ line.

5) Epigenetic marks sometimes carry over across generations and influence sexual development of opposite-sex offspring. In mammals this requires evidence that epimarks can escape the nearly genome-wide erasure that occurs while the 
primordial germ cells migrate to the genital ridge and somatic gonads, as well the nearly genome-wide erasure that occurs during the first few cleavage-stage cell cycles after syngamy.

6) Mutations that cause SA-epimarks can deterministically spread in a population.

This requires that feasible selection parameters are within the range supporting the accumulation of new mutations coding for the epimarks.

Below we evaluate these requisite conditions in mammals.

\section{Evidence for requisite conditions}

Sexually dimorphic development is canalized

Evidence for the substantial canalization of sexually dimorphic development in mammals can be found in the phenotypes in humans produced by loss-of-function mutations that influence fetal androgen signaling. In human ontogeny, as in other mammals, circulating levels of androgens are the major determinants of sexual dimorphism of the genitals and brain (summarized in Thorton et al. 2009): XX females have lower levels of fetal $\mathrm{T}$ and $\mathrm{XY}$ males have higher levels. The elevated fetal $\mathrm{T}$ in males does not occur until week 8 of human fetal development when the testes begin secreting $\mathrm{T}$.

Null mutations at the gene coding for the androgen receptor (ARnull) completely block canonical androgen signaling and cause $X Y$ fetuses to have nearly completely 
sex-reversed development, with female-typical genitalia, brains, body proportions and composition, and behavior -with the exception of the upper third of the vagina which is controlled by the absence of SRY-signaling rather than androgen-signaling (reviewed in Wisniewski et al. 2008). Note that the XY individuals (homozygous ARNull) produce substantial amounts of estrogen due to the enzymatic conversion of T secreted by their testes. These null mutations demonstrate the overarching influence of fetal androgen signaling on human sexual dimorphism.

In contrast, mutations that strongly lower or elevate T have surprisingly small effects. Null mutations in the CYP21 gene block an intermediate step in the conversion of cholesterol to cortisol. The accumulating intermediates are converted to T causing XX fetuses to experience male-typical levels of $T$ throughout fetal development (Speiser and White 2003; New 2004; Trakakis et al. 2009). Despite this male-typical phenotype for circulating fetal T, the external genitalia are only partially masculinized (Hall 2004) as is childhood behavior (Hines 2011). Although population-wide levels of transsexuality and homosexuality are weakly elevated in the subpopulation that is homozygous-null for CYP21, most individuals are female-typical for sexual identity and sexuality (reviewed in Hines 2011). The strongly feminine phenotypes produced by these loss-of-function mutations demonstrate the strong insensitivity (canalization) of $X X$ fetuses to even highly elevated levels of circulating androgens.

Null mutations in the $17 \beta-H S D-3$ locus prevent the testes from secreting $T$ during fetal development (reviewed in Rey and Grinspon 2011). Instead, the testes produce 
the precursor to $\mathrm{T}$ in the cholesterol-to-T pathway: the weakly androgenic androstenedione, which has about a hundred-fold weaker affinity for the androgen receptor (Fang et al. 2003). Despite this markedly reduced level of androgen signaling, most internal genitalia (epididymis, vas deferens, seminal vesicles, and ejaculatory duct) develop normal, or close to normal, male phenotypes. The external genitalia are substantially feminized and most affected newborns are reared as females. But at puberty, when there is a T-surge due to a non-testicular allozyme of $18 \beta-\mathrm{HSD}-3$ converting circulating, testes-produced androstenedione to $\mathrm{T}$, about half of the affected individuals change their sex to male (Wisniewski et al. 2008): the same rate as XYmales with normal circulating $\mathrm{T}$ levels throughout development that were reared as girls for other reasons (Reiner and Gearhart 2004). Although the data are limited in number, they indicate that homozygous $18 \beta-H S D-3$ Null individuals have male-typical heterosexual orientation (Imperato-McGinley et al. 1979; Meyer-Bahlburg 2005). Collectively these data indicate that $X Y$ fetuses have strong insensitivity (canalization) to even very low levels of androgen signaling.

The phenotypes of individuals with null mutations at the AR, CPY21, and 17ßHSD-3 loci provide compelling evidence that human male (XY) and female (XX) fetuses are strongly canalized with respect to even extreme sex-atypical levels of fetal androgen signaling.

A need for canalization of sexually dimorphic development 
Evidence for a fitness benefit favoring the canalization of androgen signaling described in the above section comes from studies of circulating levels of $\mathrm{T}$ in male and female fetuses. If there is substantial overlap in circulating $T$ between male and female fetuses during androgen signaling, then there will be a benefit to canalization of the androgen signal ( $X X$ embryos hypo-sensitive to $T$, and $X Y$ hypersensitive to $T$ ) because it reduces ambiguity in the androgen signal, and thereby reduces the expression of intersex phenotypes (quantitative details can be found in Rice et al. 2012). Data on humans (Reyes et al. 1974; Perera et al. 1987) and rats (Weisz and Ward 1980) demonstrate substantial overlap between the sexes in circulating levels of $T$ throughout fetal development. Despite this overlap, including the time when the external sex organs are differentiating, discordance between gonad and genitalia (phallus vs. vulva, including ambiguous genitalia) is rare, in both rats and humans (Sax 2002; Ostby et al. 1999; Hotchkiss et al. 2007).

Sex-specific epimarks produced in the embryo and early-fetal stages influence downstream sexually dimorphic development In mammals, there is epigenetic re-programming across the genome during the embryonic stem cell stage (reviewed in Hemberger et al. 2009). Thousands of genes that are expressed only late in development are silenced via DNA methylation of their promotors (Fouse et al. 2008). Many hundreds of other genes expressed in later development are bivalently marked with dominant, silencing epimarks (trimethylation of the lysine-27 residue of histone H3, i.e. H2K27) and recessive, activating epimarks (trimethylation of the lysine-4 residue of histone $\mathrm{H} 3$, i.e., $\mathrm{H} 3 \mathrm{~K} 4 \mathrm{me} 3$ ). When released 
from the suppressing H2K27 epimark, there is a strong genome-wide correlation (0.67) between the level of H3K4me3 histone modification and the level of gene expression (Mikkelsen et al. 2007).

Evidence for sex-specific epimarks during early ontogeny comes from many sources. Garner et al. (2010) surveyed evidence showing that preimplantation blastocyst embryos are sexually dimorphic far in advance of androgen signaling: including the phenotypes growth rate, metabolic rate, and resistance to several environmental stressors. Bermejo-Alvarez et al. (2010) estimated that $31 \%$ of the transcriptome is sexually dimorphic in expression level at the blastocysts stage of cattle, and in the mouse, Lowe et al. (2015) found 51 genes that were sexually dimorphically expressed in the 8 cell cleavage stage embryo (most X-linked) and 566 in the blastula stage (most autosomal). At the gene promotor level, two genes have been identified in the bovine blastocysts in which the promotor was more heavily methylated in one sex compared to the other (Bermejo-Alvarez et al. 2008; Gebert et al. 2009), and this sexspecific methylation may be influenced by differences in the expression of both DNA and histone tail methyltransferases found in the blastocysts of both cattle (BermejoAlvarez et al. 2011) and mice (Penaloza et al. 2014). Dewing et al. (2003) found XX vs. $\mathrm{XY}$ differences in gene expression in mouse embryonic brains prior to secretion of $\mathrm{T}$ by the fetal testes in $X Y$ males (51 genes, most of which were autosomal). Also in the mouse, Penaloza et al. (2014) examined the promotors of four genes with sexually dimorphic expression in the embryonic day-10.5 embryo (prior to T secretion by the testes). They found sexually dimorphic methylation of promotor CpG sites at all four 
genes, and when they reduced this dimorphism with DNA methyltransferase antagonists in cell culture, the sexual dimorphism was markedly reduced. This study also demonstrated that sexually dimorphic $\mathrm{CpG}$ methylation prior to androgen signaling was strongly associated with new sexually dimorphic $\mathrm{CpG}$ methylation produced in response to androgen signaling. Collectively, these studies indicate that the $X X$ vs. $X Y$ karyotype somehow influences (in trans) the expression of many genes during later embryo development (but before the testes start secreting $\mathrm{T}$ ) in a manner that is independent of androgen signaling, and that sexually dimorphic epimarks produced before androgen signaling can feasibly contribute to new epimarks formation later in development in response to androgen signaling.

How could such strong sexual dimorphism precede T signaling during early ontogeny? Li et al. (2014) used genome-wide CHIP/Seq to determine how many DNA locations bind SRY protein (a transcription factor) in early stage embryos (embryonic day-11.5) prior to the start of androgen signaling. They found 3,083 unique DNA binding sites. Data from Fiddler et al. (1995) in humans, and Silversides et al. (2012) in mice, demonstrate that SRY is expressed in preimplantation embryos as well as later stage fetuses (prior to androgen signaling). Data from Banovich et al. (2014) and Tsankov et al. (2015) indicate that binding to chromatin by transcription factors changes the access of these chromatin regions to both DNA and histone tail methyltransferases. Sekido (2014) has enumerated the diverse ways in which SRY can act to produce XY-specific epigenetic effects including: recruiting enzymes for DNA methylation and histone tail modifications, controlling alternative RNA splicing, regulating sex-specific miRNA levels, 
and acting as an RNA sponge that regulate transcript levels of ncRNAs. Collectively these data suggest that SRY binding may play an important role in the production of sex-specific epigenetic marks in early-stage embryos that originate far in advance of the onset of androgen signaling.

Some epigenetic marks are shared by both the soma and the germ line In the mouse model system of mammalian development, there is extensive epigenetic reprograming during the embryonic stem cell stage prior to the separation of the germ line and soma (Hemberger et al. 2009), and these epimarks can influence gene expression later in ontogeny (Mikkelsen et al. 2007). As the primordial germ cells migrate to the genital ridge, these epimarks are erased and then the genome is immediately reprogrammed with new, gamete-specific epimarks (Reik et al. 2001; LeesMurdock and Walsh 2008). One way to have a somatic SA-epimark shared between germ line and soma is a failure of the epimark to erase in the early primordial germ cell stage. Evidence for this lack of erasure comes from studies of epimarks that were environmentally induced (epimutations) in the parent (e.g., by subjecting the parent to stress or endocrine disruptors) and then carried over across one or more generations to offspring and grand-offspring. For example, hundreds of different chemically induced DNA methylation epimarks (and the phenotypes they produce) in the laboratory rat can persist through the patriline across three generations (Manikkam et al. 2012; Skinner and Guerrero-Bosagna 2014). To do so, the epimarks must persist through the broadscale epimark erasure that occurs in the primordial germ cells. These experiments demonstrate that many hundreds of epimarks in embryonic stem cells can be 
transmitted to both the soma and germ line (and are therefore shared between them), and that they can be transmitted across generations.

Epigenetic marks sometimes carry over across generations and influence sexual development of opposite-sex offspring

An epimark produced in embryonic stem cells that canalizes sexually dimorphic development will carry over transgenerationally only if it can escape erasure: i) in the early cell divisions of the primordial germ cells that give rise to the germ line, and also ii) during the nearly global erasure that occurs during the first few cleavage-stage cell divisions of the embryo. In the rodent model system, we now have evidence that it is not uncommon for methylated CpG sites to evade both erasures: more than 500 hundred chemically induced epimutations have been shown to be transmitted via the patriline for at least three generations (Manikkam et al. 2012). But can such epimarks influence sexual development in opposite sex offspring? Recent work in mice supports this outcome. Morgan and Bale (2011) exposed mothers to recurrent unpredictable episodes of mild stress (e.g., leaving the lights on all night, changing the bedding numerous times in a single day, and using damp instead of dry bedding when it was changed) during the first seven days of fetal development. Sons from these stressed mothers were feminized as measured by ano-genital distance and their sex-reversed response to a stress test. When these sons were mated to unrelated females experiencing no stress during pregnancy, their sons $\left(F_{2}\right)$ also displayed these feminized phenotypes: demonstrating transgenerational inheritance. When the transcriptome of 
the brains (PNDI region) from of these feminized sons was compared to control $F_{2}$ males and females, the sons from in utero-stressed fathers showed extensive feminization of their transcriptome -which was associated with feminization of three miRNAs with sexually dimorphic expression. This study clearly demonstrates how transgenerational epigenetic factors can strongly influence (and reverse) sexually dimorphic gene expression and development. It also illustrates the potential for a cascading effect in which a reversal in the expression of a few genes (miRNA in this case) can feasibly lead to much larger sex-reversals in the transcriptome and adult behavior.

Further evidence that carryover epimarks at one or few genes can have a large effect in reversing sexual dimorphism comes from a recent study in mice (Nugent et al. 2015). These researchers found that the preoptic area (POA) in mice becomes sexually dimorphic in response to androgen signaling during the first week of postnatal development. Sexual dimorphism in this brain area influences male mating behavior in adults. In the neonate, developing sexual dimorphism in the POA is associated with 70 genes with sexually dimorphic transcription rates. Female pups treated with simulated androgen signaling (estradiol [E2], the end-product of androgen signaling in the rodent brain) recapitulated the male phenotype, both in brain structure and adult mating behavior. Androgen signaling in both males and females resulted in strong downregulation of DNA methyltransferase (DNMT) activity and reduced the genome-wide number of sites with strongly methylated CpGs. To determine if this epigenetic (DNA methylation) effect mediated the androgen signal, they treated female pups with DNMT 
antagonists and found strong masculinization of i) the transcriptome of the POA, the POA cellular structure, and adult sexual behavior. These experiments demonstrate that the organizational effect of androgen signaling on the POA is mediated in large part by epimarks (DNA methylation induced by androgen signaling), and that it is feasible for carryover epimarks influencing one of the DNMT genes to strongly reverse a component of sexual dimorphism (brain structure and mating behavior) that is controlled by many genes simultaneously.

\section{Mutations that cause SA-epimarks can spread deterministically in a population} SA-epimarks are both beneficial to the parent that produced them and harmful to opposite-sex offspring when the epimarks carry over (un-erased) across generations and contribute to gonad-trait discordance. These costs (C) and benefits (B), however, are expected to be highly asymmetrical. The benefit to the parent producing the epimark is expressed $100 \%$ of the time, whereas the detriment is expressed only when i) the epimark carries over across generations (at rate q) and ii) it is transmitted to an opposite-sex offspring (at rate 0.5 , assuming an even sex ratio). When the transgenerational carryover rate is small, the cost benefit ratio will also be small. Previously we have solved for the conditions under which a new mutation coding for an SAepimark will accumulate in the gene pool (Rice et al. 2012). For an autosomal mutation that epimarks itself or a tightly linked location, the mutation will accumulate when $\mathrm{C} / \mathrm{B}<$ 4/q. So even when transgenerational carryover is substantial $(q=0.04)$, a mutation producing the SA epimark will accumulate whenever $\mathrm{C} / \mathrm{B}<100$. For example, a mutation that increased fitness of the parent that produced it by $1 \%$ would invade the gene pool even if it lowered the fitness of recipient opposite-sex offspring by $99 \%$. 
These calculations demonstrate that there is remarkable little selective constraint on the accumulation of mutations coding for new SA-epimarks.

Further details concerning $\mathrm{X}$ - and $\mathrm{Y}$-linkage and recombinational distance between the mutation and the epimark, as well as additional evidence for each of the other five requisite conditions, can be found in Rice et al. (2012).

\section{Discussion}

The SA-epimarks hypothesis is summarized in Figure 1. The experimental work described in the above sections provides a strong empirical foundation for the existence of SA-epimarks in nature, and the epigenetic sexual conflict they generate. Loss of function mutations in the androgen receptor (ARnull, leading to $X Y$ sex-reversed females), and enzymes in steroid biosynthesis pathways (cholesterol-to-cortisol [CYP21 null, leading to male-typical T in XX fetuses] and cholesterol-to-T [17ß-HSD-3null, leading to greatly diminished androgen levels in $\mathrm{XY}$ fetuses]) demonstrate the overarching influence of fetal androgen signaling on mammalian sexual dimorphism, and the strong canalization of male and female development (i.e., insensitivity to departures from male- and female-typical levels of fetal androgens). The experiments with cattle using sex-sorted embryos (Bermejo-Alvarez et al. 2008b; Gebert et al. 2009) clearly demonstrate that there are sex-specific epimarks and extensive sexually dimorphic gene expression in the early embryo stage -far in advance of fetal androgen signaling. Experiments on mice reinforce this conclusion in both the preimplantation embryo (Lowe et al. 2015) and the early fetal stages (Penaloza et al. 2014). Recent experiments with mice further provide evidence for a causative link between sexspecific epimarks and sexually dimorphic gene expression (Penaloza et al. 2014; 
Nugent et al. 2015) and adult phenotypes (Nugent et al. 2015). The experiments by Skinner and collaborators (e.g., Manikkam et al. 2012) on endocrine disruptors demonstrate that it is not uncommon for environmentally induced epimarks (epimutations) to escape cross-generation erasure and carry over across one or more generations. The experiments by Morgan and Bale (2011) demonstrate that stressinduced epimarks can both carry over across generations and generate substantive gonad-trait discordance. Collectively these experimental results make it plausible -we would conclude nearly inevitable- that some newly evolved, sex-specific epimarks will sometimes carry over across generations and contribute to epigenetic sexual conflict via SA-epimarks.

In mammals most sexual dimorphism is controlled by sex hormone signaling with "organization" influences during the fetal and perinatal stages by androgens and "activational" influences (that are contingent on the earlier organizational androgen signaling) that commences at puberty and are mediated by both androgens and estrogens (summarized in Thorton et al. 2009). For this reason, the most potent SAepimarks will be those that differentially influence sex hormone signaling in $X X$ vs. $X Y$ individuals. Nonetheless, SA-epimarks can be produced outside this sex hormone signaling context. As described above, mammalian embryos display sexually dimorphic phenotypes far in advance of T production by the XY fetus. Y-linked gene products, like the SRY protein, and the concentration of X-linked gene products from loci that are not dosage compensated, provide an unambiguous signal of gender that is independent of sex hormones. These XX vs. XY signals must somehow contribute to the sex-specific 
gene expression and phenotypes observed prior to the secretion of $\mathrm{T}$ by the fetal testes (as described in the preceding paragraph), and any epimarks they influence would be sexually antagonistic when they are shared between the soma and germ line and also sometimes carry over across generations and lead to discordance between the sex chromosome karyotype and gene expression.

Up to this point, we have focused on SA-epimarks originating during the embryonic stem cell (ESC) stage. The rationale for this focus was the nearly genomewide epigenetic reprogramming that occurs in ESCs, and the fact that these epimarks when unerased in early cell cycles of the primordial germ cells-are mitotically inherited by both the soma and the germ line, and hence can be shared by parent and oppositesex offspring. Franklin et al. (2010) exposed male mice ( $\left.F_{1}\right)$ to stress (prolonged separation from $\mathrm{F}_{0}$ mothers) over the first 14 days of postpartum development and observed changes in: i) behavior (a depressive-like response during a forced-swim assay), and ii) the methylation status of two genes, Crhr2 and Mecp2 (candidate genes influencing depressive-like behavior). The behavior change was observed in the $F_{1}$ males and their $F_{2}$ daughters (and their $F_{3}$ grandsons). The methylation changes were observed in both the $F_{1}$ male's sperm as well the brains of his $F_{2}$ female offspring (brains of the $F_{1}$ males were, unfortunately, not assayed). Because the stress treatment occurred after the separation of the male's soma and germ line, and because the depressive-like behavior was observed in both $F_{1}$ males and their $F_{2}$ daughters, functionally-parallel (though not necessarily identical) epigenetic changes were feasibly produced in both the soma and the germ lines and transmitted to the next generation. The transgenerational epigenetic change in the germ line was supported by the parallel 
methylation changes seen in the sperm of the $F_{1}$ males and the in the brains of their $F_{2}$ daughters. This study supports -but does not conclusively prove- that epigenetic marks can be shared between the soma and germ line when they are not mitotically inherited from the same ancestral cell(s) in which the epimark was initially produced. A possible route to this parallel-epimark-production in soma and germ line is shared miRNAs. Morgan and Bale (20011) found that miRNAs were associated with strong transgenerational feminization of the brains of sons from fathers whose mothers experienced stress during early pregnancy, and Sekido (2014) reviews evidence that the brain and gonad share miRNAs that influence somatic sexual dimorphism. If an epimarking pathway for a miRNA is shared and expressed by the soma and gonad during fetal development, parallel epimarking could lead to shared epimarks without their mitotic coalescence. While this parallel-epimark-production route to feminizing and masculinizing epimarks can clearly operate in nature, it is still unclear to us whether or not it has a substantive role in SA-epimark propagation.

SA-epimarks feasibly contribute to many features of the sexual dimorphic phenotype. One feature is the masculinity/femininity spectrum. Although the sex of most individuals is unambiguously dichotomous -male or female- many sexually dimorphic traits are distributed along a continuum between the male and female poles -with substantial overlap between the sexes. For example, human facial features have been extensively quantified by evolutionary biologists because the human face is a strong contributor to sexual attractiveness. Lee at al. (2012) used discriminant analysis of human facial landmarks to compare male and female faces. Their discriminant scores were strongly overlapping between the sexes despite a strong difference between the 
means for each sex. What causes some men to have substantially feminized faces and vice versa for women? Environmentally induced stochastic variation may account for some of the overlap, but the high heritability of masculinity/femininity of human faces (Mitchem et al. 2013) indicates that deterministic factors play a large role. Sexually antagonistic alleles could contribute to the heritability of the masculinity/femininity of human faces but theory predicts low polymorphism for SA-alleles unless they are Xlinked with strong deviations from additivity (Rice 1984) or there is a reversal of dominance between the sexes (Kidwell et al. 1977; Fry 2010, but see Arnqvist et al 2014 for the case of polygenic inheritance with epistasis). Alternatively, SA-epimarks could be the major factor contributing to the masculinization of traits like faces of females, and vice versa for males. In this case, genetic polymorphism would be unnecessary because masculinization and feminization is produced by carryover epimarks in opposite-sex offspring (even when the mutations coding for the epimarks are fixed) rather than genetic variation (Rice et al. 2012).

SA-epimarks may also feasibly contribute substantially to clinically important gonad-trait discordances like hypospadias (urethra length reduced [feminized] in males, causing a subterminal opening of the urethra along the phallus), cryptorchidism (testes position feminized due to their abdominal location), and idiopathic hirsutism (male-like body-hair pattern in women that is unassociated with hormonal imbalances). All of these gonad-trait discordances are surprisingly common. Hypospadias has a prevalence $0.4 \%$ to $1 \%$ in newborns, and can be as high as $4 \%$ when including milder cases (Boisen et al. 2005). The prevalence of cryptorchidism ranges between 2-9\% (Bay et al. 2011) and that of idiopathic hirsutism is $6 \%$ (Carmina 1998). The high incidence of these gonad- 
trait discordances is enigmatic because the two male discordances substantially reduce fertility, and the female discordance may also reduce fitness via sexual selection. No major effect genes have been found for any of these conditions despite screens with large sample sizes (see overview in Rice et al. 2012), yet recent screens -despite very small sample sizes- have found changes in both the methylome (Choudhry et al 2012) and the transcriptome (Karabulut et al 2013) of hypospadias patients. The observations described in this paragraph collectively motivate the hypothesis that SA-epimarks contribute to clinically important gonad-trait discordances.

Homosexuality represents another gonad-trait discordance. It constitutes a substantial component of humanity's sexuality spectrum, with an estimated prevalence $8 \%$ in both sexes in a large study in Australia (with homosexuality being classified as a Kinsey score > 0; Bailey et al. 2000). Homosexuality in both sexes runs in families and has higher concordance between monozygotic compared to dizygotic twins (reviewed in Ngun et al. 2011). Although early GWASs found conflicting results, a recent large GWAS found two QTLs (one X-linked and the other on autosome 8) associated male homosexuality (Sanders at al. 2015). There is also robust evidence for a birth order effect, in which males with more older brother are more likely to be homosexual (reviewed in Bogaert and Skorska 2011). In females, null mutations in the CYP21 are associated with a weak but statistically significant increase in female homosexuality (reviewed in Hines 2011). Nonetheless, the birth order effect can explain at most only only one in seven homosexual men (Cantor et al. 2002) and no homosexual women, and the two QTLs uncovered by Sanders et al. (2015) for male homosexuality have only weak effects, i.e., no genes of major effect on homosexuality have been uncovered 
despite large sampling effort in males. As we detailed elsewhere, SA-epimarks provide a feasible (Rice et al 2012), and testable (Rice et al. 2013) etiology for human homosexuality.

The information we have used to motivate and evaluate the SA-epimarks hypothesis necessarily relies heavily on studies on humans and mammalian model organisms (rodents and primates) because medical research on epigenetics far exceeds basic research with wild, non-model organisms. Nonetheless, the logical foundation for SA-epimarks in humans and mammalian model organisms should apply broadly to other taxa. The core features leading to SA-epimarks are i) sexual dimorphism and a need for its canalization, and ii) sex-specific epimarks and their transgenerational carryover. When these features are present, the potential for SAepimarks is manifest even if the specific molecular mechanisms for epigenetic marking differ among taxa. Substantial sexual dimorphism in structure and/or behavior is common among many taxa with separate sexes and a broad-scale need for canalization is indicated by the broad range of anthropogenic and naturally occurring sex steroid mimics and disruptors influencing both vertebrates and invertebrates (e.g., see Lebkowska et al. 2007). Although sex-specific epimarks are only well characterized in humans and laboratory model organisms (e.g., see examples in this paper), transgenerational carryover of epigenetic marks has been documented in taxa as diverse as plants, worms, flies, fish, birds, and mammals (Ho and Burggren 2010). These patters indicate that SA-epimarks have the potential to contribute to sexual conflict among a wide diversity of plants and animals. 
The hypothesis that SA-epimarks exist and contribute to a new and important form of sexual conflict is supported by many lines of indirect evidence (detailed above and in Rice et al. 2012) that show that all the requisite conditions exist for their adaptive advantage and their production at non-trivial frequency. Given their high feasibility, the next step will be to screen for SA-epimarks. With the advent of genome-wide screening for histone-tail modifications via CHIP/seq, and for DNA methylation of CpGs (and also $\mathrm{CHG}$ and $\mathrm{CHH}$, where $\mathrm{H}$ is $\mathrm{A}, \mathrm{C}$ or $\mathrm{T}$ ) via genome-wide bisulfate resequencing, it should now be possible to unambiguously screen for transgenerational sex-specific epimarks, determine if they occur at biologically important levels, and statistically evaluate whether they are associated with gonad-trait discordances. Ongoing screens for an epigenetic etiology of clinically important gonad-trait discordances, like cryptorchidism, hypospadias, and hirsutism, will probably be the first studies to provide the requisite data in the near future (e.g., like those recently published by Choudhry et al. 2012 and Karabulut et al. 2013). At present, we already have evidence for methylome and gene expression difference between individuals with and without hypospadias. The critical, next step in testing for SA-epimarks will be determining: i) when these epimarks are produced (in the embryonic stem cell stage or later in development, and if in later ontogeny, are they produce in parallel in both the germ line and the soma?) and the biological function of the epimarks (do they influence sexually dimorphic development and/or its canalizatiion?). At this point we can conclude that the SA-epimarks hypothesis is plausible and falsifiable, and the data needed to test this hypothesis are accessible with current technology and will be forthcoming on the near horizon. 


\section{Acknowledgements}

This work was conducted as a part of the Intragenomic Conflict Working Group at the

National Institute for Mathematical and Biological Synthesis, sponsored by the National

Science Foundation, the U.S. Department of Homeland Security, and the U.S.

Department of Agriculture through NSF Award \#EF- 0832858, with additional support

from the University of Tennessee, Knoxville. Support was also provided by the Swedish

Foundation for Strategic Research. 


\section{References}

Arnqvist G, Rowe L (1995) Sexual conflict and arms races between the sexes: a morphological adaptation for control of mating in a female insect. Proceedings of the Royal Society of London B: Biological Sciences 261, 123-127.

Arnqvist G, Rowe L (2013) Sexual Conflict Princeton University Press, New Jersey.

Arnqvist G, Vellnow N, Rowe L (2014) The effect of epistasis on sexually antagonistic genetic variation. Proceedings of the Royal Society of London B: Biological Sciences 281, 20140489.

Bailey JM, Dunne MP, Martin NG (2000) Genetic and environmental influences on sexual orientation and its correlates in an Australian twin sample. Journal of Personality and Social Psychology 78, 524-536.

Banovich NE, Lan X, McVicker G, et al. (2014) Methylation QTLs are associated with coordinated changes in transcription factor binding, histone modifications, and gene expression levels.

Bay K, Main KM, Toppari J, Skakkebæk NE (2011) Testicular descent: INSL3, testosterone, genes and the intrauterine milieu. Nature Reviews Urology 8, 187196.

Bermejo-Alvarez P, Rizos D, Lonergan P, Gutierrez-Adan A (2011) Transcriptional sexual dimorphism during preimplantation embryo development and its consequences for developmental competence and adult health and disease. Reproduction 141, 563-570. 
Bermejo-Alvarez P, Rizos D, Rath D, Lonergan P, Gutierrez-Adan A (2008) Epigenetic differences between male and female bovine blastocysts produced in vitro. Physiological Genomics 32, 264-272.

Bermejo-Alvarez P, Rizos D, Rath D, Lonergan P, Gutierrez-Adan A (2010) Sex determines the expression level of one third of the actively expressed genes in bovine blastocysts. Proceedings of the National Academy of Sciences 107, 33943399.

Bogaert AF, Skorska M (2011) Sexual orientation, fraternal birth order, and the maternal immune hypothesis: A review. Frontiers in Neuroendocrinology 32, 247-254.

Boisen K, Chellakooty M, Schmidt I, et al. (2005) Hypospadias in a cohort of 1072 Danish newborn boys: prevalence and relationship to placental weight, anthropometrical measurements at birth, and reproductive hormone levels at three months of age. The Journal of Clinical Endocrinology \& Metabolism 90, 4041-4046.

Cantor JM, Blanchard R, Paterson AD, Bogaert AF (2002) How many gay men owe their sexual orientation to fraternal birth order? Archives of Sexual Behavior 31, 63-71.

Carmina E (1998) Prevalence of idiopathic hirsutism. European journal of endocrinology $139,421-423$.

Chapman T, Liddle LF, Kalb JM, Wolfner MF, Partridge L (1995) Cost of mating in Drosophila melanogaster females is mediated by male accessory gland products. Nature 373, 241-244. 
Choudhry S, Deshpande A, Qiao L, et al. (2012) Genome-wide DNA methylation profiling of CpG islands in hypospadias. The Journal of Urology 188, 1450-1456.

Dewing P, Shi T, Horvath S, Vilain E (2003) Sexually dimorphic gene expression in mouse brain precedes gonadal differentiation. Molecular Brain Research 118, 82-90.

Fang H, Tong W, Branham WS, et al. (2003) Study of 202 natural, synthetic, and environmental chemicals for binding to the androgen receptor. Chemical Research in Toxicology 16, 1338-1358.

Fiddler M, Abdel- Rahman B, Rappolee DA, Pergament E (1995) Expression of SRY transcripts in preimplantation human embryos. American journal of medical genetics 55, 80-84.

Fouse SD, Shen Y, Pellegrini M, et al. (2008) Promoter CpG methylation contributes to ES cell gene regulation in parallel with Oct4/Nanog, PcG complex, and histone H3 K4/K27 trimethylation. Cell stem cell 2, 160-169.

Franklin TB, Russig H, Weiss IC, et al. (2010) Epigenetic transmission of the impact of early stress across generations. Biological Psychiatry 68, 408-415.

Fry JD (2010) The genomic location of sexually antagonistic variation: some cautionary comments. Evolution 64, 1510-1516.

Gavrilets S, Arnqvist G, Friberg U (2001) The evolution of female mate choice by sexual conflict. Proceedings of the Royal Society of London B: Biological Sciences 268, $531-539$. 
Gebert C, Wrenzycki C, Herrmann D, et al. (2009) DNA methylation in the IGF2 intragenic DMR is re-established in a sex-specific manner in bovine blastocysts after somatic cloning. Genomics 94, 63-69.

Guerrero-Bosagna C, Skinner MK (2014) Environmentally induced epigenetic transgenerational inheritance of male infertility. Current opinion in genetics \& development 26, 79-88.

Haldane JBS. 1926. A mathematical theory of natural and artificial selection. Part III 23, 363-372.

Hall CM, Jones JA, Meyer-Bahlburg HF, et al. (2004) Behavioral and physical masculinization are related to genotype in girls with congenital adrenal hyperplasia. The Journal of Clinical Endocrinology \& Metabolism 89, 419-424.

Hemberger M, Dean W, Reik W (2009) Epigenetic dynamics of stem cells and cell lineage commitment: digging Waddington's canal. Nature reviews Molecular cell biology 10, 526-537.

Hines M (2011) Prenatal endocrine influences on sexual orientation and on sexually differentiated childhood behavior. Frontiers in Neuroendocrinology 32, 170-182.

Ho, D. H., \& Burggren, W. W. (2010). Epigenetics and transgenerational transfer: a physiological perspective. The Journal of Experimental Biology, 213(1), 3-16.

Hotchkiss AK, Lambright CS, Ostby JS, et al. (2007) Prenatal testosterone exposure permanently masculinizes anogenital distance, nipple development, and reproductive tract morphology in female Sprague-Dawley rats. Toxicological Sciences 96, 335-345. 
Imperato-Mcginley J, Peterson RE, Gautier T, Sturla E (1979) Male

pseudohermaphroditism secondary to $5 \alpha$-reductase deficiency-a model for the role of androgens in both the development of the male phenotype and the evolution of a male gender identity. Journal of steroid biochemistry 11, 637-645.

Karabulut R, Turkyilmaz Z, Sonmez K, et al. (2013) Twenty-four genes are upregulated in patients with hypospadias. Balkan Journal of Medical Genetics 16, 39-43.

Kidwell J, Clegg M, Stewart F, Prout T (1977) Regions of stable equilibria for models of differential selection in the two sexes under random mating. Genetics 85,171 183.

Łebkowska, M., \& Załęska-Radziwiłł, M. (2007). Endocrine disruptors-ecotoxicological assays. Archiwum Ochrony Środowiska, 33, 81-88.

Lee AJ, Mitchem DG, Wright MJ, et al. (2014) Genetic factors that increase male facial masculinity decrease facial attractiveness of female relatives. Psychological Science 25, 476-484.

Lees-Murdock DJ, Walsh CP (2008) DNA methylation reprogramming in the germ line. In: Genomic Imprinting, pp. 1-15. Springer.

Li Y, Zheng M, Lau Y-FC (2014) The sex-determining factors SRY and SOX9 regulate similar target genes and promote testis cord formation during testicular differentiation. Cell Reports 8, 723-733.

Lowe R, Gemma C, Rakyan VK, Holland ML (2015) Sexually dimorphic gene expression emerges with embryonic genome activation and is dynamic throughout development. BMC genomics 16, 295. 
Mandel SPH (1971) Owens model of a genetical system with differential viability between sexes. Heredity 26, 49-63.

Manikkam M, Guerrero-Bosagna C, Tracey R, Haque MM, Skinner MK (2012)

Transgenerational actions of environmental compounds on reproductive disease and identification of epigenetic biomarkers of ancestral exposures. PloS One 7, e31901.

Meselson M, Stahl FW (1958) The replication of DNA in Escherichia coli. Proceedings of the National Academy of Sciences 44, 671-682.

Meyer-Bahlburg HF (2005) Gender identity outcome in female-raised 46, XY persons with penile agenesis, cloacal exstrophy of the bladder, or penile ablation. Archives of Sexual Behavior 34, 423-438.

Mikkelsen TS, Ku M, Jaffe DB, et al. (2007) Genome-wide maps of chromatin state in pluripotent and lineage-committed cells. Nature 448, 553-560.

Mitchem DG, Purkey AM, Grebe NM, et al. (2014) Estimating the sex-specific effects of genes on facial attractiveness and sexual dimorphism. Behavior Genetics 44, $270-281$

Morgan CP, Bale TL (2011) Early prenatal stress epigenetically programs dysmasculinization in second-generation offspring via the paternal lineage. Journal of Neuroscience 31, 11748-11755.

New MI (2004) An update of congenital adrenal hyperplasia. Annals of the New York Academy of Sciences 1038, 14-43.

Ngun TC, Ghahramani N, Sanchez FJ, Bocklandt S, Vilain E (2011) The genetics of sex differences in brain and behavior. Frontiers in Neuroendocrinology 32, 227-246. 
Nugent BM, Wright CL, Shetty AC, et al. (2015) Brain feminization requires active repression of masculinization via DNA methylation. Nature Neuroscience 18, 690-697.

Ostby J, Monosson E, Kelce WR, Gray LE (1999) Environmental antiandrogens: low doses of the fungicide vinclozolin alter sexual differentiation of the male rat. Toxicology and Industrial Health 15, 48-64.

Parker G (1979) Sexual selection and sexual conflict. In: Sexual selection and reproductive competition in insects (eds. Blum MS, Blum NA), pp. 123-166. Academic Press, New York.

Penaloza CG, Estevez B, Han DM, et al. (2014) Sex-dependent regulation of cytochrome P450 family members Cyp1a1, Cyp2e1, and Cyp7b1 by methylation of DNA. The FASEB Journal 28, 966-977.

Perera DMD, McGarrigle HHG, Lawrence DM, Lucas M (1987) Amniotic-fluid testosterone and testosterone glucuronide levels in the determination of fetal sex. Journal of Steroid Biochemistry and Molecular Biology 26, 273-277.

Reik W, Dean W, Walter J (2001) Epigenetic reprogramming in mammalian development. Science 293, 1089-1093.

Reiner WG, Gearhart JP (2004) Discordant sexual identity in some genetic males with cloacal exstrophy assigned to female sex at birth. New England Journal of Medicine 350, 333-341.

Rey RA, Grinspon RP (2011) Normal male sexual differentiation and aetiology of disorders of sex development. Best Practice \& Research Clinical Endocrinology \& Metabolism 25, 221-238. 
Reyes FI, Borodits RS, Winter JSD, Faiman C (1974) Studies on human sexual development. 2. Fetal and maternal serum gonadotropin and sex steroid concentrations. Journal of Clinical Endocrinology \& Metabolism 38, 612-617.

Rice WR (1984) Sex chromosomes and the evolution of sexual dimorphism. Evolution, 735-742.

Rice WR (1996) Sexually antagonistic male adaptation triggered by experimental arrest of female evolution. Nature 381, 232-234.

Rice WR, Friberg U, Gavrilets S (2012) Homosexuality as a consequence of epigenetically canalized sexual development. Quarterly Review of Biology 87, 343-368.

Rice WR, Friberg U, Gavrilets S (2013) Homosexuality via canalized sexual development: a testing protocol for a new epigenetic model. Bioessays 35, 764770.

Rice WR, Holland B (1997) The enemies within: intergenomic conflict, interlocus contest evolution (ICE), and the intraspecific Red Queen. Behavioral Ecology and Sociobiology 41, 1-10.

Rice WR, Gavrilets S (2014) The Genetics and Biology of Sexual Conflict Cold Spring Harbor Laboratory press, Cold Spring Harbor, New York.

Sanders A, Martin E, Beecham G, et al. (2015) Genome-wide scan demonstrates significant linkage for male sexual orientation. Psychological Medicine 45, 13791388.

Sax L (2002) How common is intersex? A response to Anne Fausto- Sterling. Journal of Sex Research 39, 174-178. 
Sekido R (2014) The potential role of SRY in epigenetic gene regulation during brain sexual differentiation in mammals. Advances in Genetics 86, 135-165.

Silversides DW, Raiwet DL, Souchkova O, Viger RS, Pilon N (2012) Transgenic mouse analysis of Sry expression during the pre- and peri- implantation stage. Developmental Dynamics 241, 1192-1204.

Skinner MK, Guerrero-Bosagna C (2014) Role of CpG deserts in the epigenetic transgenerational inheritance of differential DNA methylation regions. BMC Genomics 15, 692.

Speiser PW, White PC (2003) Congenital adrenal hyperplasia. New England Journal of Medicine 349, 776-788.

Thornton J, Zehr JL, Loose MD (2009) Effects of prenatal androgens on rhesus monkeys: A model system to explore the organizational hypothesis in primates. Hormones and Behavior 55, 633-644.

Trakakis E, Loghis C, Kassanos D (2009) Congenital adrenal hyperplasia because of 21-hydroxylase deficiency: A genetic disorder of interest to obstetricians and gynecologists. Obstetrical \& gynecological survey 64, 177-189.

Tsankov AM, Gu H, Akopian V, et al. (2015) Transcription factor binding dynamics during human ES cell differentiation. Nature 518, 344-349.

Watson JD, Crick FHC (1953a) Molecular structure of nucleic acids. Nature 171, 737738.

Watson JD, Crick FHC (1953b) Genetic implications of the structure of deoxyribonucleic acid. Nature 171, 964-967. 
Weisz J, Ward IL (1980) Plasma testosterone and progesterone titers of pregnant rats, their male and female fetuses, and neonatal offspring. Endocrinology 106, 306316.

Wisniewski AB, Kirk KD, Copeland KC (2008) Long-term psychosexual development in genetic males affected by disorders of sex development (46, XY DSD) reared male or female. Current Pediatric Reviews 4, 243-249. 
Figure 1. Sexually antagonistic epigenetic marks (SA-epimarks) occur when a sexspecific epimark -that canalizes sexually dimorphic phenotypes during later ontogenyis produced in the embryonic stem cell stage (ESC) and fails to erase during early germ line differentiation and also during early early embryonic development in opposite-sex offspring. The transgenerationally inherited, sex-specific epimark contributes to gonadtrait discordance in the next generation. SA epimarks can also arise from sex-specific epimarks produced during later development when they are produced in parallel in both the soma and the germ line. 


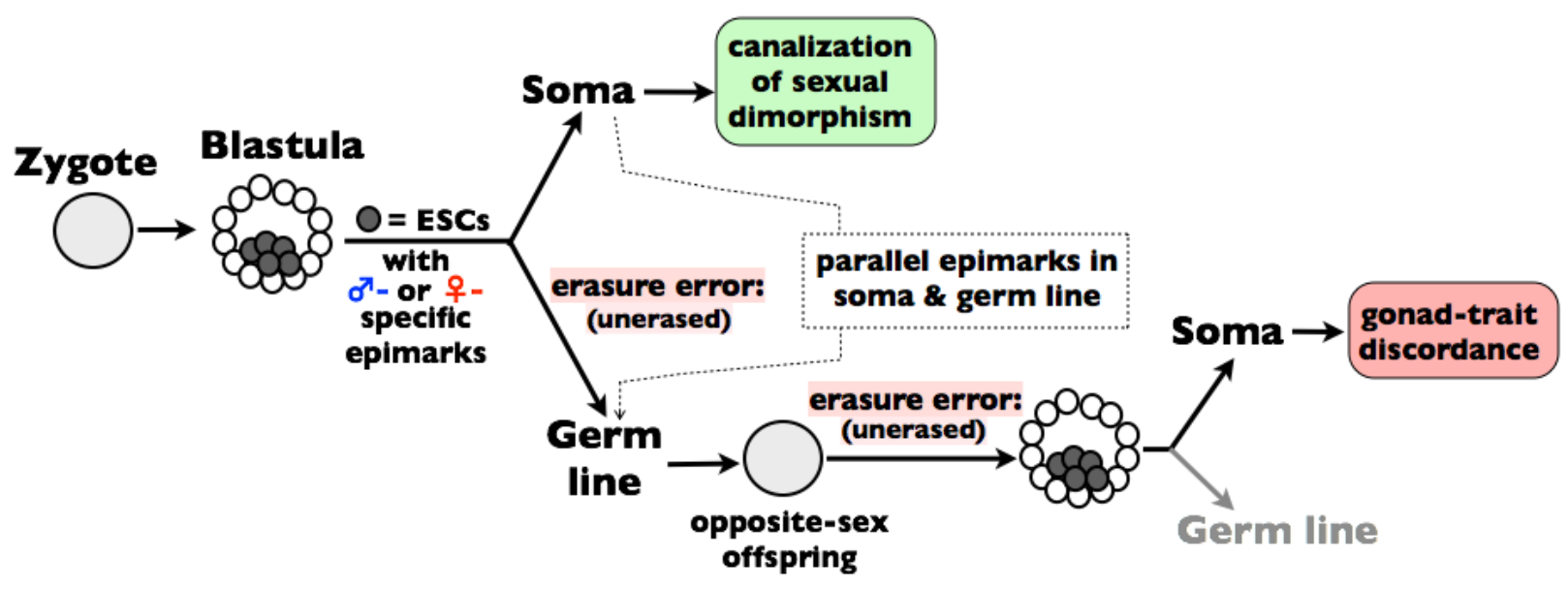

Figure I. Sexually antagonistic epigenetic marks (SA-epimarks) occur when a sex-specific epimark -that canalizes sexually dimorphic phenotypes during later ontogeny- is produced in the embryonic stem cell stage (ESC) and fails to erase during early germ line differentiation and also during early early embryonic development in opposite-sex offspring. The transgeneranally inherited, sex-specific epimark contributes to gonad-trait discordance in the next generation. SA epimarks can also arise from sex-specific epimarks produced during later development when they are produced in parallel in both the soma and the germ line. 